\title{
ON THE STRONG LAW OF LARGE NUMBERS FOR WEIGHTED SUMS OF NEGATIVELY SUPERADDITIVE DEPENDENT RANDOM VARIABLES
}

\author{
Aiting Shen
}

\begin{abstract}
Let $\left\{X_{n}, n \geq 1\right\}$ be a sequence of negatively superadditive dependent random variables. In the paper, we study the strong law of large numbers for general weighted sums $\frac{1}{g(n)} \sum_{i=1}^{n} \frac{X_{i}}{h(i)}$ of negatively superadditive dependent random variables with non-identical distribution. Some sufficient conditions for the strong law of large numbers are provided. As applications, the Kolmogorov strong law of large numbers and Marcinkiewicz-Zygmund strong law of large numbers for negatively superadditive dependent random variables are obtained. Our results generalize the corresponding ones for independent random variables and negatively associated random variables.
\end{abstract}

\section{Introduction}

As is well known that the Kolmogorov strong law of large numbers and Marcinkiewicz-Zygmund strong law of large numbers play important roles in probability limit theory and mathematical statistics, which have been studied by many authors. It is more interesting to consider a general case. Let $g(x)$ and $h(x)$ be positive functions defined on $(0, \infty)$ such that $g(x)$ is strictly increasing and $\lim _{x \rightarrow \infty} g(x)=\infty$. It is very desirable to investigate conditions on $g, h$ and random variables $\left\{X_{n}, n \geq 1\right\}$ under which the strong law of large numbers for weighted sums

$$
\frac{1}{g(n)} \sum_{i=1}^{n} \frac{X_{i}}{h(i)} \rightarrow 0 \text { a.s., as } n \rightarrow \infty
$$

Received April 14, 2014; Revised July 8, 2015.

2010 Mathematics Subject Classification. 60F15.

Key words and phrases. negatively superadditive dependent random variables, Marcinkiewicz-Zygmund strong law of large numbers, weighted sums, the three series theorem.

Supported by the National Natural Science Foundation of China (11201001, 11501004, 11501005), the Natural Science Foundation of Anhui Province (1508085J06, 1308085QA03), the Provincial Natural Science Research Project of Anhui Colleges (KJ2015A018), the Open Project of School of Mathematical Sciences, Anhui University (ADSY201503), the Quality Improvement Projects for Undergraduate Education of Anhui University (ZLTS2015035) and the Students Innovative Training Project of Anhui University (201510357116). 
holds. It is easily seen that the strong law of large numbers of the form (1.1) embraces the Kolmogorov strong law of large numbers $(g(n)=n, h(n)=1)$ and the Marcinkiewicz-Zygmund strong law of large numbers $\left(g(n)=n^{1 / r}\right.$, $h(n)=1,1<r<2)$.

Recently, Jajte [5] gave the sufficient and necessary conditions for (1.1) based on independent and identically distributed random variables. The main result of Jajte [5] is as follows.

Theorem A. Let $\left\{X_{n}, n \geq 1\right\}$ be a sequence of independent and identically distributed random variables. Let $g(\cdot)$ be a positive, increasing function and $h(\cdot)$ a positive function such that $\phi(y) \equiv g(y) h(y)$ satisfies the following conditions.

(i) For some $d \geq 0, \phi(\cdot)$ is strictly increasing on $[d, \infty)$ with range $[0, \infty)$.

(ii) There exist $C$ and a positive integer $k_{0}$ such that $\phi(y+1) / \phi(y) \leq C$ for all $y \geq k_{0}$.

(iii) There exist constants $a$ and $b$ such that for all $s>d$,

$$
\phi^{2}(s) \int_{s}^{\infty} \frac{1}{\phi^{2}(x)} d x \leq a s+b .
$$

Then the following two conditions are equivalent:

(1) $E\left[\phi^{-1}\left(\left|X_{1}\right|\right)\right]<\infty$,

(2)

$$
\frac{1}{g(n)} \sum_{i=1}^{n} \frac{X_{i}-m_{i}}{h(i)} \rightarrow 0 \text { a.s., as } n \rightarrow \infty,
$$

where $m_{i}=E X_{i} I\left(\left|X_{i}\right| \leq \phi(i)\right)$ and $\phi^{-1}$ is the inverse of function $\phi$.

Inspired by Jajte [5], Jing and Liang [6] extended the result of Jajte [5] to negatively associated random variables with identical distribution. Meng and Lin [9], Wang [20] and Tang [13] extended Theorem A to the case of $\tilde{\rho}$-mixing random variables, non-identically distributed negatively associated random variables and asymptotically almost negatively associated random variables, respectively. Sung [12] gave some sufficient conditions to prove the strong law of large numbers for weighted sums of random variables. The main purpose of the paper is to extend the result of Theorem A to the case of negatively superadditive dependent random variables, which contains independent random variables and negatively associated (NA, in short) random variables as special cases. The main techniques used in the paper are the Khintchine-Kolmogorov type convergence theorem and the Kolmogorov three series theorem.

Now, let us recall the concept of negatively superadditive dependent random variables, which was introduced by $\mathrm{Hu}[4]$.

Definition 1.1 (cf. Kemperman [8]). A function $\phi: \mathbb{R}^{n} \rightarrow \mathbb{R}$ is called superadditive if $\phi(\mathbf{x} \vee \mathbf{y})+\phi(\mathbf{x} \wedge \mathbf{y}) \geq \phi(\mathbf{x})+\phi(\mathbf{y})$ for all $\mathbf{x}, \mathbf{y} \in \mathbb{R}^{n}$, where $\vee$ is for componentwise maximum and $\wedge$ is for componentwise minimum. 
Definition 1.2 (cf. Hu, [4]). A random vector $\mathbf{X}=\left(X_{1}, X_{2}, \ldots, X_{n}\right)$ is said to be negatively superadditive dependent (NSD) if

$$
E \phi\left(X_{1}, X_{2}, \ldots, X_{n}\right) \leq E \phi\left(X_{1}^{*}, X_{2}^{*}, \ldots, X_{n}^{*}\right)
$$

holds for every superadditive function $\phi$ such that the expectations in (1.2) exist, where $X_{1}^{*}, X_{2}^{*}, \ldots, X_{n}^{*}$ are independent such that $X_{i}^{*}$ and $X_{i}$ have the same distribution for each $i$.

A sequence $\left\{X_{n}, n \geq 1\right\}$ of random variables is said to be NSD if for all $n \geq 1,\left(X_{1}, X_{2}, \ldots, X_{n}\right)$ is NSD.

The concept of NSD random variables was introduced by $\mathrm{Hu}$ [4], which was based on the class of superadditive functions. $\mathrm{Hu}$ [4] gave an example illustrating that NSD does not imply negative association (NA, in sort, see Joag-Dev and Proschan [7]), and Hu posed an open problem whether NA implies NSD. Christofides and Vaggelatou [1] solved this problem and indicated that NA implies NSD. By the Property $P_{2}$ in Hu [4], we can see that NSD random variables are negatively orthant dependent (NOD, in short). For more details about NOD random variables, one can refer to Joag-Dev and Proschan [7] and Wang et al. [16, 17], Wu [21], Wu and Jiang [22], and so forth. Negatively superadditive dependent structure is an extension of negatively associated structure and sometimes more useful than it and can be used to get many important probability inequalities. Eghbal et al. [2] derived two maximal inequalities and strong law of large numbers of quadratic forms of NSD random variables under the assumption that $\left\{X_{i}, i \geq 1\right\}$ is a sequence of nonnegative NSD random variables with $E X_{i}^{r}<\infty$ for all $i \geq 1$ and some $r>1$. Eghbal et al. [3] provided some Kolmogorov inequality for quadratic forms $T_{n}=\sum_{1 \leq i<j \leq n} X_{i} X_{j}$ and weighted quadratic forms $Q_{n}=\sum_{1 \leq i<j \leq n} a_{i j} X_{i} X_{j}$, where $\left\{X_{i}, i \geq 1\right\}$ is a sequence of nonnegative NSD uniformly bounded random variables. Shen et al. [11] studied the almost sure convergence theorem and strong stability for weighted sums of NSD random variables. Wang et al. [15] obtained complete convergence for arrays of rowwise NSD random variables and gave application in nonparametric regression models. Shen et al. [10] presented some applications of the Rosenthal-type inequality for NSD random variables. Wang et al. [18] established some general results on complete convergence for weighted sums of NSD random variables and gave its application in the EV regression model. Wang and $\mathrm{Hu}[14]$ investigated the strong consistency of $M$-estimates in linear models for NSD errors, and so forth. In this work, we will further study the strong law of large numbers for weighted sums (1.1) of NSD random variables.

Finally, we will present the concept of stochastic domination, which will be used in this paper.

Definition 1.3. A sequence $\left\{X_{n}, n \geq 1\right\}$ of random variables is said to be stochastically dominated by a random variable $X$ if there exists a positive 
constant $C$ such that

$$
P\left(\left|X_{n}\right|>x\right) \leq C P(|X|>x)
$$

for all $x \geq 0$ and $n \geq 1$.

The organization of this paper is as follows: main results are presented in Section 2. Some important lemmas and the proofs of the main results are provided in Section 3 and Section 4, respectively.

Throughout the paper, let $C$ denote a positive constant not depending on $n$, which may be different in various places. Let $\lfloor x\rfloor$ denote the integer part of $x$ and $I(A)$ be the indicator function of the set $A$.

\section{Main results}

In this section, let $\left\{X_{n}, n \geq 1\right\}$ be a sequence of NSD random variables, which is stochastically dominated by a random variable $X$. Let

$$
m_{i}=E X_{i} I\left(\left|X_{i}\right| \leq \phi(i)\right)
$$

and $\phi^{-1}$ is the inverse of function $\phi$, where $\phi$ is defined in Theorem 2.1. Firstly, we will extend the sufficient part of Theorem A to the NSD setting.

Theorem 2.1. Let $g(\cdot)$ and $h(\cdot)$ be real positive functions defined on the same domain $(0, \infty)$ such that $\phi(y) \equiv g(y) h(y)$ satisfies conditions of (i) and (iii) in Theorem A. If $E\left[\phi^{-1}(|X|)\right]<\infty$, then

$$
\sum_{n=1}^{\infty} \frac{X_{n}-m_{n}}{\phi(n)} \text { converges a.s.. }
$$

If we further assume that $g(x)$ is increasing on its domain, and $\lim _{x \rightarrow \infty} g(x)=$ $\infty$, then

$$
\frac{1}{g(n)} \sum_{i=1}^{n} \frac{X_{i}-m_{i}}{h(i)} \rightarrow 0 \text { a.s., as } n \rightarrow \infty .
$$

In the following, we will provide some sufficient conditions to prove the strong law of large numbers for the weighted sums (1.1).

Theorem 2.2. Let $g(\cdot)$ and $h(\cdot)$ be real positive functions defined on the same domain $(0, \infty)$ such that $\phi(y) \equiv g(y) h(y)$ satisfies the conditions of (i) and (iii) in Theorem A. If $E X_{n}=0$ and $E\left[\phi^{-1}(|X|)\right]<\infty$, then

$$
\sum_{n=1}^{\infty} \frac{X_{n}}{\phi(n)} \text { converges a.s.. }
$$

If we further assume that $g(x)$ is increasing on its domain, and $\lim _{x \rightarrow \infty} g(x)=$ $\infty$, then

$$
\frac{1}{g(n)} \sum_{i=1}^{n} \frac{X_{i}}{h(i)} \rightarrow 0 \quad \text { a.s., as } n \rightarrow \infty \text {. }
$$


Theorem 2.3. Let $g(\cdot)$ and $h(\cdot)$ be real positive functions defined on the same domain $(0, \infty)$ such that $\phi(y) \equiv g(y) h(y)$ satisfies the conditions of (i) and (iii) in Theorem A. Suppose that

$$
\int_{r}^{\infty} \frac{1}{\phi(x)} d x \leq \operatorname{lr} / \phi(r) \text { for some } r \geq 1 \text { and } l>0 .
$$

If $E\left[\phi^{-1}(|X|)\right]<\infty$, then (2.3) holds. If we further assume that $g(x)$ is increasing on its domain, and $\lim _{x \rightarrow \infty} g(x)=\infty$, then (2.4) holds.

By Theorem 2.2, we can get the Kolmogorov strong law of large numbers and Marcinkiewicz-Zygmund strong law of large numbers for NSD random variables as follows.

Corollary 2.1. Let $\left\{X_{n}, n \geq 1\right\}$ be a sequence of identically distributed $N S D$ random variables. If $E\left|X_{1}\right|^{p}<\infty$ for some $1 \leq p<2$, then

$$
\frac{1}{n^{1 / p}} \sum_{i=1}^{n}\left(X_{i}-E X_{1}\right) \rightarrow 0 \text { a.s., as } n \rightarrow \infty \text {. }
$$

If we take $g(x)=\log x$ and $h(x)=x$ in Theorem 2.2 , then we can get the following particular case of logarithmic means.

Corollary 2.2. Let $\left\{X_{n}, n \geq 1\right\}$ be a sequence of mean zero NSD random variables, which is stochastically dominated by a random variable $X$. If $E|X|<$ $\infty$, then

$$
\frac{1}{\log n} \sum_{k=1}^{n} \frac{X_{k}}{k} \rightarrow 0 \quad \text { a.s., as } n \rightarrow \infty \text {. }
$$

\section{Some useful lemmas}

In this section, we will present some important lemmas which will be used to prove the main results of the paper. The first one is a basic property for NSD random variables, which was given by $\mathrm{Hu}[4]$.

Lemma 3.1 (cf. Hu, [4]). Let $\left(X_{1}, X_{2}, \ldots, X_{n}\right)$ be NSD.

(i) $\left(-X_{1},-X_{2}, \ldots,-X_{n}\right)$ is also $N S D$.

(ii) If $g_{1}, g_{2}, \ldots, g_{n}$ are all nondecreasing functions, then $\left(g_{1}\left(X_{1}\right), g_{2}\left(X_{2}\right)\right.$, $\left.\ldots, g_{n}\left(X_{n}\right)\right)$ is $N S D$.

$\mathrm{Hu}$ [4] established a comparison theorem on moments between the NSD and independent random variables and pointed out that the Rosenthal maximal inequality remains true for NSD random variables. By using the Rosenthal maximal inequality, we can get the following Khintchine-Kolmogorov type convergence theorem and Kolmogorov three series theorem for NSD random variables immediately. The proofs are standard, so we omit the details.

Lemma 3.2 (Khintchine-Kolmogorov type convergence theorem). Let $\left\{X_{n}\right.$, $n \geq 1\}$ be a sequence of NSD random variables. Assume that $\sum_{n=1}^{\infty} \operatorname{Var}\left(X_{n}\right)<$ $\infty$. Then $\sum_{n=1}^{\infty}\left(X_{n}-E X_{n}\right)$ converges a.s.. 
Lemma 3.3 (Kolmogorov three series theorem). Let $\left\{X_{n}, n \geq 1\right\}$ be a sequence of NSD random variables. Denote $X_{n}^{(c)}=-c I\left(X_{n}<-c\right)+X_{n} I\left(\left|X_{n}\right| \leq c\right)+$ $c I\left(X_{n}>c\right)$, where $c$ is a positive constant. If the following three conditions are satisfied:

(i) $\sum_{n=1}^{\infty} P\left(\left|X_{n}\right|>c\right)<\infty$;

(ii) $\sum_{n=1}^{\infty} E X_{n}^{(c)}$ converges;

(iii) $\sum_{n=1}^{\infty} \operatorname{Var}\left(X_{n}^{(c)}\right)<\infty$, then $\sum_{n=1}^{\infty} X_{n}$ converges a.s..

By the definition of stochastic domination and integration by parts, we can get the following basic inequalities. The proof is standard, so we omit the details. One can refer to $\mathrm{Wu}$ [21] or Wang et al. [19] for instance.

Lemma 3.4. Let $\left\{X_{n}, n \geq 1\right\}$ be a sequence of random variables which is stochastically dominated by a random variable $X$. For any $\alpha>0$ and $b>0$, the following two statements hold:

$$
\begin{gathered}
E\left|X_{n}\right|^{\alpha} I\left(\left|X_{n}\right| \leq b\right) \leq C_{1}\left[E|X|^{\alpha} I(|X| \leq b)+b^{\alpha} P(|X|>b)\right], \\
E\left|X_{n}\right|^{\alpha} I\left(\left|X_{n}\right|>b\right) \leq C_{2} E|X|^{\alpha} I(|X|>b),
\end{gathered}
$$

where $C_{1}$ and $C_{2}$ are positive constants. Consequently, $E\left|X_{n}\right|^{\alpha} \leq C E|X|^{\alpha}$.

\section{Proofs of the main results}

Proof of Theorem 2.1. Denote for $n \geq 1$ that

$$
Y_{n}=-\phi(n) I\left(X_{n}<-\phi(n)\right)+X_{n} I\left(\left|X_{n}\right| \leq \phi(n)\right)+\phi(n) I\left(X_{n}>\phi(n)\right) .
$$

By the definition of stochastic domination and the condition $E\left[\phi^{-1}(|X|)\right]<$ $\infty$, we can see that

$$
\begin{aligned}
\sum_{n=1}^{\infty} P\left(X_{n} \neq Y_{n}\right) & =\sum_{n=1}^{\infty} P\left(\left|X_{n}\right|>\phi(n)\right) \\
& \leq C \sum_{n=1}^{\infty} P(|X|>\phi(n)) \\
& =C \sum_{n=1}^{\infty} P\left(\phi^{-1}(|X|)>n\right) \\
& \leq C E\left[\phi^{-1}(|X|)\right]<\infty .
\end{aligned}
$$

Combining (4.1) and Borel-Cantelli lemma, we have

$$
P\left(\left(X_{n} \neq Y_{n}\right) \text {, i.o. }\right)=0 \text {. }
$$


Next, we will consider the series $\sum_{n=1}^{\infty} \frac{E Y_{n}^{2}}{\phi^{2}(n)}$. By $C_{r}$ 's inequality, definition of stochastic domination, Lemma 3.4 and (4.1), we can get that

$$
\begin{aligned}
\sum_{n=1}^{\infty} \frac{E Y_{n}^{2}}{\phi^{2}(n)} & \leq C \sum_{n=1}^{\infty} \frac{1}{\phi^{2}(n)}\left[E X_{n}^{2} I\left(\left|X_{n}\right| \leq \phi(n)\right)+\phi^{2}(n) P\left(\left|X_{n}\right|>\phi(n)\right)\right] \\
& \leq C \sum_{n=1}^{\infty} \frac{E X^{2} I(|X| \leq \phi(n))}{\phi^{2}(n)}+C \sum_{n=1}^{\infty} P(|X|>\phi(n)) \\
& \leq C E\left[\sum_{n=1}^{\infty} \frac{X^{2} I(|X| \leq \phi(n))}{\phi^{2}(n)}\right] .
\end{aligned}
$$

Since $E\left[\phi^{-1}(|X|)\right]<\infty$, it follows that $\phi^{-1}(|X|)<\infty$ a.s. and $\phi\left(\phi^{-1}(|X|)\right)=$ $|X|$. Hence, we have by the condition (iii) in Theorem $\mathrm{A}$ that

$$
\begin{aligned}
& \sum_{n=1}^{\infty} \frac{X^{2} I(|X| \leq \phi(n))}{\phi^{2}(n)} \\
= & \sum_{n=1}^{\left\lfloor\phi^{-1}(|X|)\right\rfloor+1} \frac{X^{2} I(|X| \leq \phi(n))}{\phi^{2}(n)}+\sum_{\left\lfloor\phi^{-1}(|X|)\right\rfloor+2}^{\infty} \frac{X^{2} I(|X| \leq \phi(n))}{\phi^{2}(n)} \\
\leq & \left\lfloor\phi^{-1}(|X|)\right\rfloor+1+\sum_{\left\lfloor\phi^{-1}(|X|)\right\rfloor+2}^{\infty} \frac{X^{2}}{\phi^{2}(n)} \\
\leq & \phi^{-1}(|X|)+1+X^{2} \int_{\phi^{-1}(|X|)}^{\infty} \frac{1}{\phi^{2}(x)} d x \\
\leq & (1+a) \phi^{-1}(|X|)+1+b,
\end{aligned}
$$

which implies that

$$
E\left[\sum_{n=1}^{\infty} \frac{X^{2} I(|X| \leq \phi(n))}{\phi^{2}(n)}\right] \leq(1+a) E\left[\phi^{-1}(|X|)\right]+1+b<\infty .
$$

By (4.3) and (4.4), we have

$$
\sum_{n=1}^{\infty} \frac{\operatorname{Var}\left(Y_{n}\right)}{\phi^{2}(n)} \leq \sum_{n=1}^{\infty} \frac{E Y_{n}^{2}}{\phi^{2}(n)}<\infty
$$

It is easily seen that $\left\{Y_{n} / \phi(n), n \geq 1\right\}$ is also a sequence of NSD random variables by Lemma 3.1. Hence, we have by (4.5) and Lemma 3.2 that

$$
\sum_{n=1}^{\infty} \frac{Y_{n}-E Y_{n}}{\phi(n)} \text { converges a.s.. }
$$

Combining (4.2) and (4.6), we can get that

$$
\sum_{n=1}^{\infty} \frac{X_{n}-E Y_{n}}{\phi(n)} \text { converges a.s.. }
$$


To complete the proof of (2.1), we only need to show that

$$
\begin{aligned}
& \sum_{n=1}^{\infty} \frac{\phi(n) P\left(X_{n}<-\phi(n)\right)-\phi(n) P\left(X_{n}>\phi(n)\right)}{\phi(n)} \\
= & \sum_{n=1}^{\infty}\left[P\left(X_{n}<-\phi(n)\right)-P\left(X_{n}>\phi(n)\right)\right] \text { converges. }
\end{aligned}
$$

Note that

$$
\sum_{n=1}^{\infty} P\left(\left|X_{n}\right|>\phi(n)\right)=\sum_{n=1}^{\infty} P\left(X_{n}<-\phi(n)\right)+\sum_{n=1}^{\infty} P\left(X_{n}>\phi(n)\right)<\infty,
$$

and $\sum_{n=1}^{\infty} P\left(X_{n}<-\phi(n)\right)$ and $\sum_{n=1}^{\infty} P\left(X_{n}>\phi(n)\right)$ are both nonnegative, it follows that $\sum_{n=1}^{\infty} P\left(X_{n}<-\phi(n)\right)$ and $\sum_{n=1}^{\infty} P\left(X_{n}>\phi(n)\right)$ are both convergent, which yield (4.7). This completes the proof of (2.1). Finally, $\frac{1}{g(n)} \sum_{i=1}^{n} \frac{X_{i}-m_{i}}{h(i)} \rightarrow 0$ a.s. follows from (2.1) and Kronecker's lemma immediately. The proof is complete.

Proof of Theorem 2.2. Denote for $n \geq 1$ that

$$
\begin{aligned}
Z_{n} & =-I\left(X_{n} / \phi(n)<-1\right)+\frac{X_{n}}{\phi(n)} I\left(\left|X_{n} / \phi(n)\right| \leq 1\right)+I\left(X_{n} / \phi(n)>1\right) \\
& \doteq\left(\frac{X_{n}}{\phi(n)}\right)^{(1)} .
\end{aligned}
$$

We will apply Lemma 3.3 to the sequence $\left\{X_{n} / \phi(n), n \geq 1\right\}$, where $c=1$. Here, $\left\{X_{n} / \phi(n), n \geq 1\right\}$ is still a sequence of NSD random variables by Lemma 3.1 .

By the definition of stochastic domination and the condition $E\left[\phi^{-1}(|X|)\right]<$ $\infty$, we can see that

$$
\begin{aligned}
\sum_{n=1}^{\infty} P\left(\left|X_{n}\right| / \phi(n)>1\right) & \leq C \sum_{n=1}^{\infty} P(|X|>\phi(n)) \\
& =C \sum_{n=1}^{\infty} P\left(\phi^{-1}(|X|)>n\right) \\
& \leq C E\left[\phi^{-1}(|X|)\right]<\infty
\end{aligned}
$$

Similar to the proof of (4.3)-(4.5), we can get that

$$
\begin{aligned}
\sum_{n=1}^{\infty} \operatorname{Var}\left(Z_{n}\right) & \leq \sum_{n=1}^{\infty} E Z_{n}^{2} \\
& \leq C \sum_{n=1}^{\infty}\left[\frac{1}{\phi^{2}(n)} E X_{n}^{2} I\left(\left|X_{n}\right| \leq \phi(n)\right)+P\left(\left|X_{n}\right|>\phi(n)\right)\right] \\
& <\infty .
\end{aligned}
$$


Finally, we will show that

$$
\sum_{n=1}^{\infty} E Z_{n} \text { converges. }
$$

By (4.8) and Borel-Cantelli lemma, we have

$$
P\left(\left\{\left|X_{n}\right| / \phi(n)>1\right\}, \text { i.o. }\right)=0
$$

and

$$
\sum_{n=1}^{\infty} \frac{\left|X_{n}\right|}{\phi(n)} I\left(\left|X_{n}\right| / \phi(n)>1\right)<\infty \text { a.s. }
$$

which imply that

$$
\sum_{n=1}^{\infty} E\left|X_{n} / \phi(n)\right| I\left(\left|X_{n}\right| / \phi(n)>1\right)<\infty .
$$

Thus, we have by $E X_{n}=0,(4.8)$ and (4.11) that

$$
\begin{aligned}
\sum_{n=1}^{\infty}\left|E Z_{n}\right| & \leq \sum_{n=1}^{\infty} P\left(\left|X_{n}\right| / \phi(n)>1\right)+\sum_{n=1}^{\infty} E\left|X_{n} / \phi(n) I\left(\left|X_{n}\right| / \phi(n) \leq 1\right)\right| \\
& \leq \sum_{n=1}^{\infty} P\left(\left|X_{n}\right| / \phi(n)>1\right)+\sum_{n=1}^{\infty} E\left|X_{n} / \phi(n)\right| I\left(\left|X_{n}\right| / \phi(n)>1\right) \\
& <\infty .
\end{aligned}
$$

Therefore, the desired result (2.3) follows from (4.8), (4.9), (4.12) and Lemma 3.3 immediately, and (2.4) follows (2.3) and Kronecker's lemma. This completes the proof of the theorem.

Proof of Theorem 2.3. We use the same notations as that in Theorem 2.2. According to the proof of Theorem 2.2, we can see that in order to prove $(2.3)$ and (2.4), we only need to show (4.10). Actually, by (2.5), (4.8) and Lemma 3.4 , we have

$$
\begin{aligned}
\sum_{n=1}^{\infty}\left|E Z_{n}\right| & \leq C \sum_{n=1}^{\infty} \frac{1}{\phi(n)} E|X| I(|X| \leq \phi(n))+C \sum_{n=1}^{\infty} P(|X|>\phi(n)) \\
& \leq C \sum_{n=1}^{\infty} \frac{1}{\phi(n)} \sum_{j=1}^{n} E|X| I\left(j-1<\phi^{-1}(|X|) \leq j\right) \\
& \leq C \sum_{n=1}^{\infty} \frac{1}{\phi(n)} \sum_{j=1}^{n} \phi(j) P\left(j-1<\phi^{-1}(|X|) \leq j\right) \\
& =C \sum_{j=1}^{\infty} \phi(j) P\left(j-1<\phi^{-1}(|X|) \leq j\right) \sum_{n=j}^{\infty} \frac{1}{\phi(n)}
\end{aligned}
$$




$$
\begin{aligned}
& \leq C \sum_{j=1}^{\infty} \phi(j) P\left(j-1<\phi^{-1}(|X|) \leq j\right)\left[\frac{1}{\phi(j)}+\int_{j}^{\infty} \frac{1}{\phi(x)} d x\right] \\
& \leq C \sum_{j=1}^{\infty} j P\left(j-1<\phi^{-1}(|X|) \leq j\right) \\
& \leq C \sum_{n=1}^{\infty} P\left(\phi^{-1}(|X|) \geq n\right) \\
& \leq C E\left[\phi^{-1}(|X|)\right]<\infty .
\end{aligned}
$$

This completes the proof of the theorem.

Proof of Corollary 2.1. Without loss of generality, we assume that $E X_{1}=0$. Thus, in order to prove (2.6), we only need to show that

$$
\frac{1}{n^{1 / p}} \sum_{i=1}^{n} X_{i} \rightarrow 0 \text { a.s., as } n \rightarrow \infty \text {. }
$$

Take

$$
\begin{aligned}
& g(x)=x^{1 / p}, 0<p<2, x \in(0, \infty) \\
& h(x)=1, x \in(0, \infty) \\
& \phi(x)=g(x) h(x), x \in(0, \infty), \phi(0)=0 .
\end{aligned}
$$

It is easily seen that the conditions of Theorem 2.2 are satisfied. Hence, the desired result (4.13) follows from Theorem 2.2 immediately. This completes the proof of the corollary.

Acknowledgements. The authors are most grateful to the Editor and anonymous referee for careful reading of the manuscript and valuable suggestions which helped in improving an earlier version of this paper.

\section{References}

[1] T. C. Christofides and E. Vaggelatou, A connection between supermodular ordering and positive/negative association, J. Multivariate Anal. 88 (2004), no. 1, 138-151.

[2] N. Eghbal, M. Amini, and A. Bozorgnia, Some maximal inequalities for quadratic forms of negative superadditive dependence random variables, Statist. Probab. Lett. 80 (2010), no. $7-8,587-591$.

[3] - On the Kolmogorov inequalities for quadratic forms of dependent uniformly bounded random variables, Statist. Probab. Lett. 81 (2011), no. 8, 1112-1120.

[4] T. Z. Hu, Negatively superadditive dependence of random variables with applications, Chinese J. Appl. Probab. Statist. 16 (2000), no. 2, 133-144.

[5] R. Jajte, On the strong law of large numbers, Ann. Probab. 31 (2003), no. 1, 409-412.

[6] B. Y. Jing and H. Y. Liang, Strong limit theorems for weighted sums of negatively associated random variables, J. Theoret. Probab. 21 (2008), no. 4, 890-909.

[7] K. Joag-Dev and F. Proschan, Negative association of random variables with applications, Ann. Statist. 11 (1983), no. 1, 286-295.

[8] J. H. B. Kemperman, On the FKG-inequalities for measures on a partially ordered space, Nederl. Akad. Wetensch. Proc. Ser. A 80 (1977), no. 4, 313-331. 
[9] Y. J. Meng and Z. Y. Lin Strong laws of large numbers for $\tilde{\rho}$-mixing random variables, J. Math. Anal. Appl. 365 (2010), no. 2, 711-717.

[10] A. T. Shen, Y. Zhang, and A. Volodin, Applications of the Rosenthal-type inequality for negatively superadditive dependent random variables, Metrika 78 (2015), no. 3, 295-311.

[11] Y. Shen, X. J. Wang, W. Z. Yang, and S. H. Hu, Almost sure convergence theorem and strong stability for weighted sums of NSD random variables, Acta Math. Sin. English Series 29 (2012), no. 4, 743-756.

[12] S. H. Sung, On the strong law of large numbers for weighted sums of random variables, Comput. Math. Appl. 62 (2011), no. 11, 4277-4287.

[13] X. F. Tang, Some strong laws of large numbers for weighted sums of asymptotically almost negatively associated random variables, J. Inequal. Appl. 2013 (2013), Article ID 4, 11 pages.

[14] X. H. Wang and S. H. Hu, On the strong consistency of $M$-estimates in linear models for negatively superadditive dependent errors, Aust. New Zealand J. Stat. 57 (2015), no. $2,259-274$

[15] X. J. Wang, X. Deng, L. L. Zheng, and S. H. Hu, Complete convergence for arrays of rowwise negatively superadditive dependent random variables and its applications, Statistics 48 (2014), no. 4, 834-850.

[16] X. J. Wang, S. H. Hu, A. Shen, and W. Z. Yang, An exponential inequality for a NOD sequence and a strong law of large numbers, Appl. Math. Lett. 24 (2011), no. 2, 219-223.

[17] X. J. Wang, S. H. Hu, and W. Z. Yang, Complete convergence for arrays of rowwise negatively orthant dependent random variables, RACSAM 106 (2012), no. 2, 235-245.

[18] X. J. Wang, A. T. Shen, Z. Y. Chen, and S. H. Hu, Complete convergence for weighted sums of NSD random variables and its application in the EV regression model, TEST 24 (2015), no. 1, 166-184.

[19] X. J. Wang, C. Xu, T.-C. Hu, A. Volodin, and S. H. Hu, On complete convergence for widely orthant-dependent random variables and its applications in nonparametric regression models, TEST 23 (2014), no. 3, 607-629.

[20] Z. Z. Wang, On strong law of large numbers for dependent random variables, J. Inequal. Appl. 2011 (2011), Article ID 279754, 13 pages.

[21] Q. Y. Wu, A complete convergence theorem for weighted sums of arrays of rowwise negatively dependent random variables, J. Inequal. Appl. 2012 (2012), Article ID 50, 10 pages.

[22] Q. Y. Wu and Y. Y. Jiang, The strong consistency of $M$ estimator in a linear model for negatively dependent random samples, Comm. Statist. Theory Methods 40 (2011), no. $3,467-491$.

School of Mathematical Sciences

AnHui University

Hefei 230601, P. R. China

E-mail address: Empress201010@126.com 